\title{
High-fat diet induces an ovulatory defect associated with dysregulated endothelin-2 in mice
}

\author{
Natalie M Hohos ${ }^{1}$, Emily M Elliott ${ }^{1}$, Asma Giornazi ${ }^{1}$, Elena Silva ${ }^{1}$, John D Rice ${ }^{2}$ and \\ Malgorzata E Skaznik-Wikiel ${ }^{3}$ \\ ${ }^{1}$ Division of Reproductive Sciences, Department of Obstetrics and Gynecology, University of Colorado School of \\ Medicine, Aurora, Colorado, USA, ${ }^{2}$ Department of Biostatistics and Informatics, Colorado School of Public Health, \\ Aurora, Colorado, USA and ${ }^{3}$ Division of Reproductive Endocrinology and Infertility, Department of Obstetrics and \\ Gynecology, University of Colorado School of Medicine, Aurora, Colorado, USA
}

Correspondence should be addressed to M E Skaznik-Wikiel; Email: malgorzata.skaznik-wikiel@cuanschutz.edu

\begin{abstract}
High-fat diet (HFD) consumption in female rodents causes impaired estrous cyclicity, fewer pups per litter, and dysregulation of key ovulatory genes suggesting that HFD-induced subfertility may be due to ovulatory dysfunction. To test this hypothesis female mice were fed chow or HFD for 10 weeks at which point ovulation and ovarian gene expression of endothelin-2 (Edn2), a gene critical for ovulation, were assessed. After 10 weeks of HFD, both mice that remained lean and those that became obese had fewer ovulated oocytes than chow controls $(P=0.041, P=0.0030$, respectively). In chow controls, Edn2 was expressed as expected with basal levels during diestrus and proestrus, increased 11.6-fold during estrus, and decreased to basal levels during metestrus. In HFD mice, Edn2 was dysregulated across the entire estrous cycle as were other Edn2 system components (endothelin converting enzyme 1 (Ece-1), and the endothelin receptors (Ednra, Ednrb)). Interestingly, we found dysregulation of key ovarian steroidogenic genes after HFD. We also found that estradiol treatment in prepubertal mice increased $E d n 2$ expression in the ovary $(P=0.030)$, suggesting that impaired steroidogenesis may be involved in the HFD-induced dysregulation of ovarian Edn2. In conclusion, HFD leads to ovulatory dysfunction regardless of the development of obesity, which appears to be mediated through dysregulation of ovarian Edn2 expression.
\end{abstract}

Reproduction (2021) $161307-317$

\section{Introduction}

High-fat diet (HFD) consumption in female rodents leads to ovarian dysfunction and subfertility regardless of the development of the obese phenotype (SkaznikWikiel et al. 2016). HFD feeding has also been shown to result in impaired estrous cyclicity (Hohos et al. 2018) and fewer pups per litter in consecutive breeding attempts (Skaznik-Wikiel et al. 2016), suggesting that the HFD-induced subfertile phenotype may in part be due to ovulatory dysfunction. Interestingly, it has been shown that HFD-fed obese mice have fewer ovulated oocytes than chow-fed controls (Ge et al. 2014), however, it is unknown if this ovulatory defect is present without diet-induced obesity. In order to gain a better understanding of the ovarian changes leading to the subfertile phenotype induced by HFD, we examined ovarian transcriptomic changes in response to HFD and identified several ovulatory genes that were dysregulated after HFD feeding, regardless of the development of obesity (Hohos et al. 2018), further strengthening the idea that ovulatory dysfunction is playing a role in HFDinduced subfertility. In our discovery approach, we identified one dysregulated ovulatory gene of particular interest, endothelin-2 (gene Edn2, protein ET2).

ET2 plays a critical role in ovulation (Migone et al. 2016). Edn2's expression has been shown to be markedly and acutely increased immediately prior to ovulation in the granulosa cells of preovulatory follicles of superovulated rodents (Ko et al. 2006, Na et al. 2008, Bridges et al. 2010). This striking increase in ET2 induces contraction of the smooth muscle cells in the follicular wall of the pre-ovulatory follicle, allowing the wall to rupture and the oocyte to be released (Migone et al. 2016, Cacioppo et al. 2017). When ET2's actions are blocked prior to ovulation there are unruptured follicles present in the ovary and fewer ovulated oocytes (Ko et al. 2006, Palanisamy et al. 2006), all of which can be rescued with the administration of the ET2 peptide (Cacioppo et al. 2014). Although this relationship between ovarian ET2 and ovulation has been well documented in superovulated rodent models, it has yet to be confirmed in naturally cycling mice. Further, ET2 levels were decreased in PCOS-induced rat ovaries, however, treatment of PCOS with DMBG for 4 weeks was able to increase ET2 expression, but not to the 
level of controls (Wang et al. 2015). Edn2, as well as the other components of the endothelin system, have been shown to be present in human granulosa cells and the endothelin receptors have been shown to be present in the smooth muscle cells of human ovarian tissue, suggesting that ET2 may also be important in human ovulatory function (Choi et al. 2011). Work in bovine models suggests that $E d n 2$ may also be important in angiogenesis involved in corpus luteum formation (Klipper et al. 2010), implying another role for Edn2 in the ovary, however, this has yet to be confirmed in other species. Edn2 positive cells have also been identified in the ovarian stroma, capsule, granulosa cells of small and large follicles, and in the corpus luteum in the mouse (Cacioppo et al. 2015), suggesting that there may be other roles for $E d n 2$ outside of ovulation in the ovary.

Once the endothelin-2 peptide is produced in the ovary it undergoes several processing steps to get to the bioactive form, ET2. The peptide that is produced from Edn2 is the Prepro-ET-2, which is converted to Big-ET2 by a furin-like protease and then is converted to ET2 by either endothelin converting enzyme 1 or 2 (ECE-1, ECE-2, respectively) (Ling et al. 2013), however, in the ovary it has been suggested that ECE- 1 is involved in ET2 processing (Bridges et al. 2010). ECE-1 expression has been shown to be reduced by LH (Levy et al. 2003, Shimizu et al. 2007) and upregulated by IGF-1 and insulin in bovine granulosa cells. The lowest levels of ECE- 1 were found in the early stages of bovine $\mathrm{CL}$ development and as the CL matured ECE-1 levels increased and remained elevated in the late CL (Levy et al. 2003). Choudhary et al. reported that the activity of ECE-1 remained unchanged throughout the ovarian cycle in bovine models. ECE-1 gene was constitutively expressed until the final phase of the lifespan of the $\mathrm{CL}$, when the gene expression decreased (Choudhary et al. 2004). ECE-1 production has also been shown to be negatively affected by prostaglandin F2alpha in very early pregnancy in ewes (Costine et al. 2007), however, prostaglandin F2alpha treatment had no effect on ECE-1 activity during early-, mid-, and late- luteal phase in bovines (Choudhary et al. 2004).

The mechanisms regulating Edn2 expression in the ovary are complex. Cell culture of granulosa cells have shown that both $\mathrm{LH}$ and hypoxia increase Edn2 expression (Klipper et al. 2010). It has also been shown that Hif1a, a hypoxia factor, is a transcription factor for Edn2 expression ( $\mathrm{Na}$ et al. 2008, Kim et al. 2009, Wang et al. 2012, Zhang et al. 2012, Yalu et al. 2015). Hif1a expression on the other hand is induced in a progesterone receptor-dependent manner, particularly in the granulosa cells of the preovulatory follicles (Kim et al. 2009).

In the current study, we aimed to determine the possible role of ovarian Edn2 in HFD-induced ovulatory dysfunction. We first determined if there is an ovulatory defect induced by HFD in HFD-fed mice that remain lean, similar to what has been shown in obese HFD-fed mice.
We then explored the role of ovarian Edn2 in the context of the observed ovulatory defect. Since the levels of Edn2 in naturally cycling mice have yet to be characterized, we began our work by characterizing ovarian Edn2 expression across the estrous cycle in naturally cycling chow-fed mice and then investigated the impact of HFD.

\section{Materials and methods}

\section{Animals and diet}

All mice (C57BL/6) female mice obtained from Jackson Laboratories) were housed at the Anschutz Medical Campus Center for Comparative Medicine facilities. The University of Colorado Anschutz Medical Campus Institutional Animal Care and Use Committee approved all mouse procedures (protocol\#00126). Mice were housed four-five per cage with free access to food and water (ad libitum feeding), as previously described (Skaznik-Wikiel et al. 2016). Mice were examined daily for wellness, signs of cage aggression, and disease.

Five-week-old C57BL/6J mice were assigned to receive either a chow diet (Teklad 2920X, Envigo, Madison, WI) containing 15.1\% calories from fat (ovulation study: $n=12$, chow; Edn2 across estrous cycle study: $n=37$, chow) or a HFD (D12492, Research Diets, New Brunswick, NJ) containing $60 \%$ of calories from fat (ovulation study: $n=36$, HFD; Edn2 across estrous cycle study $n=36$, HFD) as previously described (Hohos et al. 2018). Mice were maintained on their respective diets for 10 weeks.

For the mice in the ovulation study, after 10 weeks of diet, HFD-fed mice were separated into three tertiles based on body weight: the lightest tertile designated HFD-lean (HFLn, $n=12$, $\leq 26 \mathrm{~g}$ ), the middle tertile excluded (26-30 g, $n=12$ ), and the heaviest tertile designated HFD-obese (HFOb, $n=12$, >30 g). Chow, HFLn and HFOb mice in the ovulation study were then superovulated with an intraperitoneal (IP) injection of 5IU of pregnant mare serum gonadotropin followed $48 \mathrm{~h}$ later by an IP injection of 5IU of human chorionic gonadotropin (hCG).

\section{Evaluation of estrous cycle}

After 8 weeks of dietary intervention vaginal smears were collected daily from mice and vaginal cytology was used to determine the estrous cycle stage as previously described (Caligioni 2009, Hohos et al. 2018). To determine if mice were cycling normally at least 14 consecutive days were assessed. A mouse was determined to be cycling normally if they exhibited correct stage progression, appropriate cycle length (3-5 days), and/or no extended period in any stage ( $>3$ days). All mice were included in the analysis regardless of their estrous cyclicity status.

\section{Tissue collection}

For the ovulation study, mice were sacrificed $20 \mathrm{~h}$ after hCG injection, fasted 2-4 $\mathrm{h}$ prior to sacrifice. Before sacrifice, body composition was determined by quantitative magnetic resonance (Echo-MRI-900 Whole Body Composition Analyzer, Echo Medical Systems, Houston, TX). Blood was then collected 
from the tail veil and glucose levels were determined with the ReliOn blood glucose monitoring system (Ultima). Mice were then sacrificed and blood was immediately collected via cardiac puncture and serum was collected from blood as previously described (Hohos et al. 2018). The ovaries and oviducts were then removed and placed in $37^{\circ} \mathrm{CM} 2$ medium (cat\# SLCC2812, Sigma) and ovulated cumulus-oocyte complexes (COC) were collected by puncturing each oviduct under a dissecting microscope. The ovulated COCs were then denuded of cumulus cells with $0.1 \%$ hyaluronidase (cat\# H4272, Sigma) in M2 Medium and oocytes were then classified by their developmental stage (Germinal Vesicle (GV) - visible GV, Metaphase I (MI)-no visible polar body or GV, Metaphase II (MII)-visible extruded polar body (Mao et al. 2014)) or by their fragmentation status. All steps working with oocytes were performed at $37^{\circ} \mathrm{C}$. The remaining ovaries were cleaned and fixed in $4 \%$ paraformaldehyde, embedded in paraffin, and sectioned.

For the studies examining the effect of HFD on ovarian Edn2 across the estrous cycle, to ensure at least $n=8$ mice could be collected in each of the four estrous cycle stages in each diet group, cycling of mice continued into the 10th week of diet until at least an $n=8$ mice in each estrous stage in each diet group were obtained. This resulted in a final sample size of $n=8$ diestrus-chow, $n=9$ proestrus-chow, $n=11$ estrus-chow, $n=9$ metestrus-chow, $n=9$ diestrus-HFD, $n=8$ proestrusHFD, $n=10$ estrus-HFD, and $n=9$ metestrus-HFD. Prior to sacrifice, body composition was determined by quantitative magnetic resonance and blood was then collected from the tail veil and glucose levels were determined after at least a $2 \mathrm{~h}$ fast. Mice were then euthanized and blood was immediately collected via cardiac puncture and serum was collected from blood as previously described (Hohos et al. 2018). The ovaries were collected, immediately flash-frozen in liquid Nitrogen and stored at $-80^{\circ} \mathrm{C}$. RNA, DNA, and protein were extracted from the ovary using the Allprep DNA/RNA/Protein mini kit following manufacture protocols (cat\# 80004, Qiagen).

\section{Serum hormones}

Serum levels of follicle-stimulating hormone $(\mathrm{FSH})$ and luteinizing hormone $(\mathrm{LH})$ were determined with the Milliplex Mouse Pituitary Magnetic Bead Panel (cat\#MPTMAG-49K,
Millipore Sigma) following manufacturer's protocols from the mice in the Edn2 across the estrous cycle study that were in diestrus and estrus. For FSH, the assay's sensitivity was $9.50 \mathrm{pg} /$ $\mathrm{mL}$ with an intra-assay CV of $<15 \%$ and an inter-assay CV of $<20 \%$. For $\mathrm{LH}$, the assay's sensitivity was $1.90 \mathrm{pg} / \mathrm{mL}$ with an intra-assay CV of $<15 \%$ and an inter-assay CV of $<20 \%$.

\section{qRT-PCR}

Ovarian RNA quality and quantity were determined with the Nanodrop 2000 Spectrophotometer (Thermo Scientific). In total, 400 ng of RNA was used for cDNA synthesis using the qScript cDNA synthesis supermix (cat\# 95048-100, Quanta Biosciences, Beverly, MA). Oligonucleotide primer sequences were determined for 13 genes (Edn2, Ece1, Esr1, Esr2, Cyp11a1, Cyp19a1, Ednra, Ednrb, Hsd17b, Star, Plin2, Bactin and 18s) and synthesized by Integrated DNA Technologies (Coralville, IA) (Table 1). A $10 \mu \mathrm{L}$ reaction using PowerUp SYBR green MasterMix (cat\# A5742, Applied Biosystems) and 2 ng of cDNA was performed following manufacture protocols. Either $18 \mathrm{~s}$ or Bactin was used as endogenous controls. Relative quantitates (RQ) were determined for each gene relative to the chow mice or to the diestrus stage if compared within diet using the ddCT method (Livak \& Schmittgen 2001).

\section{Lipid and hematoxylin and eosin (H\&E) ovarian staining}

Ovaries from chow and HFD-fed mice were rehydrated and stained with anti-Perilipin-2 (Plin2) antibody (cat\# 20RAP002, Fitzgerald, Acton, MA) and counterstained with Harris Hematoxylin. Negative controls underwent the same staining but with no primary antibody.

\section{Statistical analysis}

All statistical analyses were conducted using GraphPad Prism 8 software. Data were first tested for normality using Kolmogorov-Smirnov and Shapiro-Wilk tests. Normally distributed continuous data were analyzed using Student's t-test or one-way ANOVA, followed by Tukey's post hoc test. For continuous data that did not follow normal distribution,

Table 1 Oligonucleotides for each transcript assayed are presented.

\begin{tabular}{|c|c|c|}
\hline \multirow[b]{2}{*}{ Genes } & \multicolumn{2}{|c|}{ Oligonucleotide sequences $\left(5^{\prime}-3^{\prime}\right)$} \\
\hline & Sense & Anti-sense \\
\hline$E d n 2$ & TAGCTACTGTACCTGCTTGGA & GTGGGATTGCTCCTGGATTT \\
\hline Ece1 & CTGACTAGAAGCCAACACCATAG & САСTGGACGTCTATCTGGAATG \\
\hline Esr1 & GAGATTATGCCTGGGAAGGAAA & AAAGAACCСАСТGАСССАТTAG \\
\hline Esr2 & GTAGAACGGTGTGGTCATCAA & CTGTGAGGTAGGAATGCGAAA \\
\hline Cyp11a1 & ACATCCAGGCCAACATTAC & TCAAGTTGTGTGCCATCTC \\
\hline Cyp19a1 & GTCCATCAAGCAGCATTTG & CCAGGAAGAGCATGTTAGAG \\
\hline Ednra & GCGTGAGACCAACATAAG & CGGATTGTCAGCCATTAC \\
\hline Ednrb & CTGATGACCTGCGAAATG & CCAACAGAGAGCAAACAC \\
\hline$H s d 17 b$ & GTGCACACAGCCTTCTAT & CTCAGGGCTTGCTCATAAC \\
\hline Star & TCCСTTATCGTGTGGGTAA & CACCGAGGTGAGCAATAAA \\
\hline Plin2 & CAACAGAGCGTGGTGATGA & CGGGTACTGATCCTTTGTACTG \\
\hline Actb & GACGTTGACATCCGTAAAG & GGAGCCAGAGCAGTAAT \\
\hline $18 s$ & CGGCTTAATTTGACTCAACAC & ATCAATCTGTCAATCCTGTCC \\
\hline
\end{tabular}


we used Mann-Whitney test. Categorical data were analyzed using Fischer's exact test. All data are presented as mean \pm S.D., unless otherwise specified. Differences were considered to be statistically significant at $P<0.05$.

\section{Results}

\section{Superovulation study}

After 10 weeks of HFD, HFOb mice weighed more than both chow and HFLn mice (Fig. $1 \mathrm{~A}, P<0.05$ ). There was no difference in body weight between the HFLn mice and chow-fed controls (Fig. 1A). Percent body fat was increased in the HFOb mice compared to the chow and HFLn mice (Fig. 1B, $P<0.05$ ). The HFLn mice had a
A

\section{Body Weight}

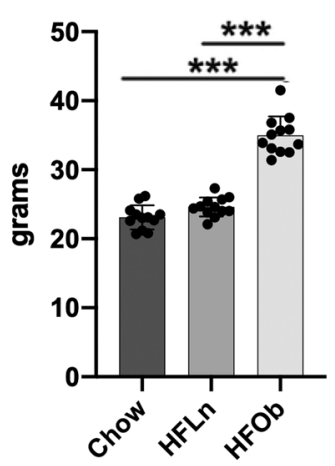

C

Blood Glucose

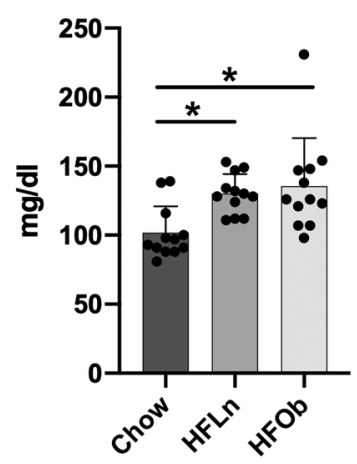

B

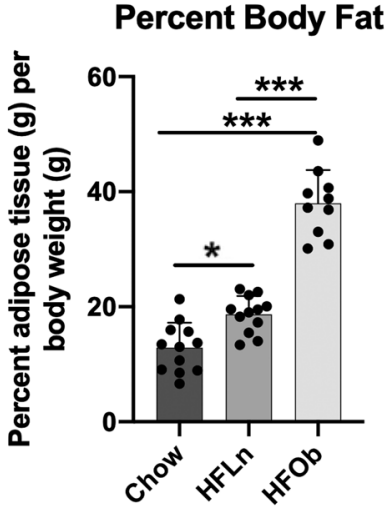

D

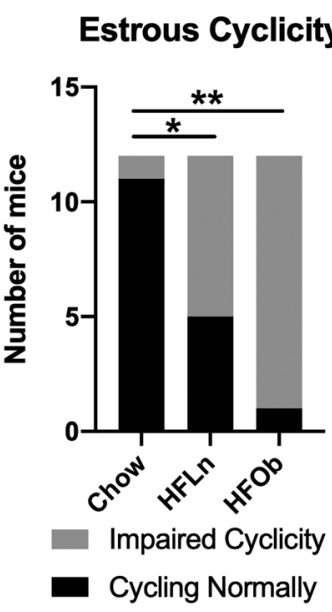

Figure 1 Ovulation study anthropometrics and estrous cyclicity. Anthropometric (A and B) and metabolomic (C) parameters were measured in mice after 10 weeks of HFD-exposure. Data are presented as means \pm S.D. Differences between groups were determined with a one-way ANOVA followed by Tukey's post hoc test if the overall ANOVA reached statistical significance. Daily vaginal cytology was assessed for the last 2 weeks of dietary feeding to evaluate the estrous cycle (D). Differences in estrous cyclicity between groups were determined by Fischer's exact test. ${ }^{*} P<0.05$; ${ }^{* *} P<0.005 ;{ }^{* * *} P<0.0005 . n=12$ /group. higher percent of body fat than chow controls, however, this was still much lower than the percent body fat of the $\mathrm{HFOb}$ mice (Fig. 1B, $P<0.05$ ). Both HFLn and HFOb had increased gene and protein expression of the lipid droplet protein Plin2, a proxy for lipid content (Yang et al. 2010), in their ovaries compared to the chowfed controls (Fig. 2, $P<0.05$ ). Fasting blood glucose values were elevated in both the HFOb and HFLn mice compared to the chow controls (Fig. 1C, $P<0.05$ ). There was a higher prevalence of mice with impaired estrous cyclicity in both the HFOb (11/12) and HFLn $(7 / 12)$ groups compared to the chow-fed controls $(1 / 12)$ (Fig. 1D, $P<0.05$ ).

An ovulatory defect was present in both HFOb and HFLn mice after 10 weeks of HFD, with HFOb mice only ovulating $18.00 \pm 7.87$ oocytes and HFLn mice only ovulating $13.67 \pm 9.52$ oocytes compared to the $28.58 \pm 12.62$ oocytes ovulated by the chow-fed controls (Fig. 3A, $P<0.05$ ). Although the HFLn mice ovulated fewer oocytes than the chow-fed controls,

A

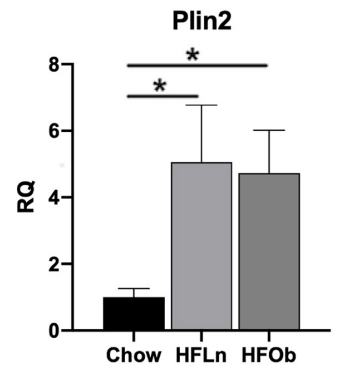

B
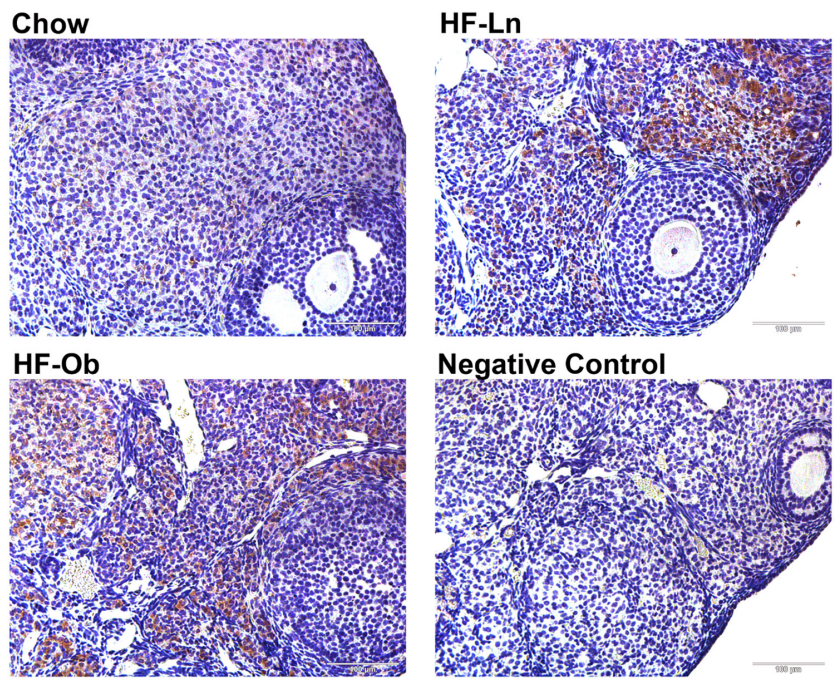

Figure 2 Ovarian lipid deposition. After 10 weeks of HFD-feeding, ovarian Plin2 levels were measured (A). Data are presented as means \pm S.D. Differences between groups were determined by a one-way ANOVA followed by Tukey's post hoc test if the overall ANOVA reached statistical significance. Representative images of ovarian anti-Plin2 staining are shown in (B). ${ }^{*} P<0.05$. Chow, $n=15$; HFLn, $n=10$; HFOb, $n=9$. Publicly available DOI for Figshare data: 10.6084/m9.figshare.12170100. 
they had a similar percentage of ovulated MII oocytes (Fig. 3B). HFOb mice however, had a lower percentage of MII ovulated oocytes compared to the chow-fed controls (Fig. 3B, $P<0.05$ ). There was no difference in the percent of ovulated $\mathrm{MI}$ or GV oocytes between either of the HFD groups and the chow controls (Fig. $3 \mathrm{C}$ and D). A higher percentage of fragmented oocytes was detected in the HFLn mice compared to the chowfed controls $(P<0.05)$. Although the HFOb mice had a higher percentage of fragmented oocytes compared to the chow-fed controls, this did not reach statistical significance $(P=0.12)$ (Fig. $3 \mathrm{E})$.

\section{Effect of HFD on ovarian Edn2 expression across the estrous cycle}

As we detected that HFD induces an ovulatory defect (fewer ovulated oocytes) and have previously reported dysregulation of ovarian Edn2 gene expression in diestrus after HFD feeding (Hohos et al. 2018), we proceeded to determine if dysregulation of ovarian Edn2 could be involved in the observed ovulatory defect. Comparisons presented are between the total HFD group (not HFLn and HFOb subsets), and controls, as the same ovulatory defect (fewer ovulated oocytes) was observed in HFLn and HFOb mice.

After 10 weeks of HFD feeding, HFD-fed mice in all estrous cycle stages weighed more and had a higher percent body fat than chow-fed controls (Fig. 4A and B,
$P<0.05)$. HFD mice in estrus and metestrus had higher blood glucose levels then their respective chow fed controls (Fig. 4C, $P<0.05$ ). A significant effect of estrous stage on blood glucose was observed, with higher blood glucose levels in proestrus than estrus regardless of dietary exposure $(P<0.05)$.

After 10 weeks of HFD, 3/9 (33\%) mice in the HFD diestrus group had normal estrous cycles compared to $5 / 8(62 \%)$ mice in the chow diestrus group, $4 / 8(50 \%)$ mice in the HFD proestrus group had normal estrous cycles compared to $8 / 9(89 \%)$ mice in the chow proestrus group, $3 / 10(30 \%)$ mice in the HFD estrus group had normal estrous cycles compared to $9 / 11(81 \%)$ mice in the chow estrus group, and 4/9 (44\%) mice in the HFD metestrus group had normal estrous cycles compared to $7 / 9(77 \%)$ mice in the chow metestrus group (Fig. 4D).

No significant differences were observed between serum FSH levels in either diestrus or estrus between the chow and HFD-fed mice (Fig. 4E). Similarly, there were no differences between serum $\mathrm{LH}$ levels in either diestrus or estrus between the chow and HFD-fed mice (Fig. 4F).

We first examined Edn2 ovarian expression levels across the estrous cycle within each diet. As Edn2 expression has been shown to have peak levels around ovulation in superovulated mice (Ko et al. 2006, $\mathrm{Na}$ et al. 2008, Bridges et al. 2010), we expected to observe basal levels of Edn2 in diestrus and proestrus, a sharp rise in estrus, and a return to basal levels in
A

Ovulated Oocytes

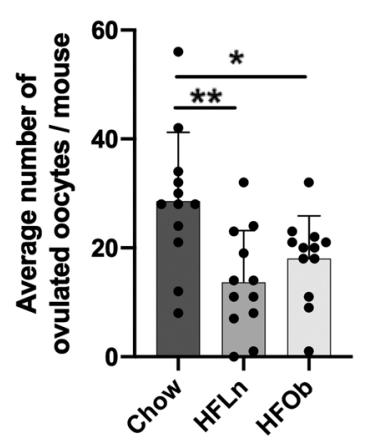

D

GV Oocytes

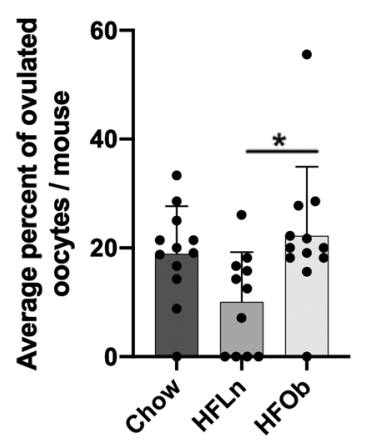

$B$

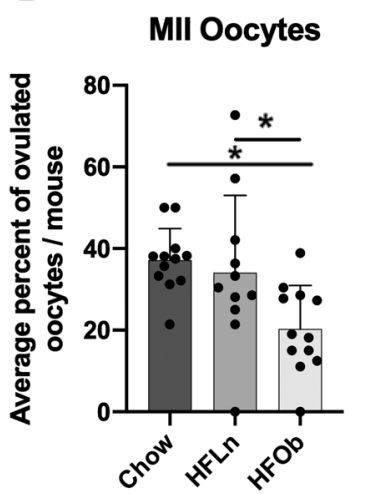

E

Fragmented Oocytes

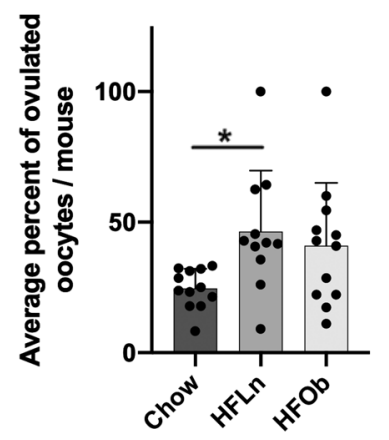

C

\section{Oocytes}

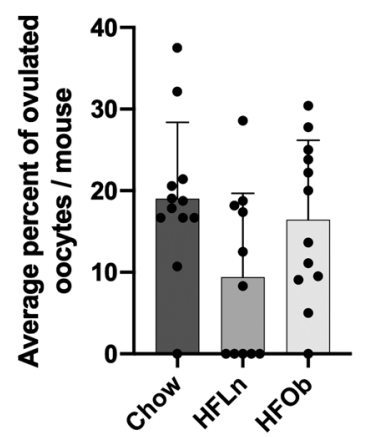

https://rep.bioscientifica.com

Reproduction (2021) 161 307-317

Figure 3 Impact of HFD on ovulation and oocyte developmental status. The number of ovulated oocytes/mouse was determined after 10 weeks of dietary intervention (A). The developmental quality (A, B, C and D) or the fragmentation status (E) of the ovulated oocytes were classified per group. Data are presented as means \pm S.D. Differences between groups was determined with a one-way ANOVA followed by Tukey's post hoc test if the overall ANOVA reached statistical significance. ${ }^{*} P<0.05 ;{ }^{*} P<0.005 . n=12$ per group. 
A

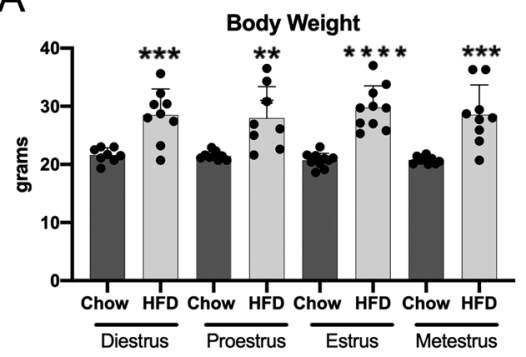

D

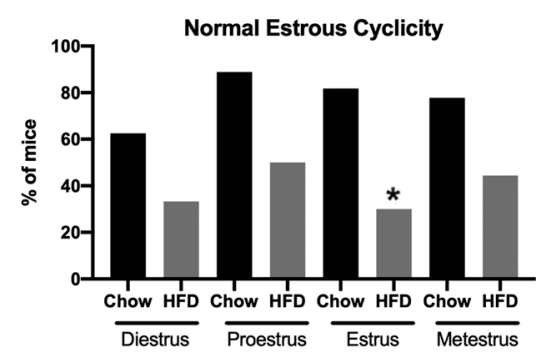

B

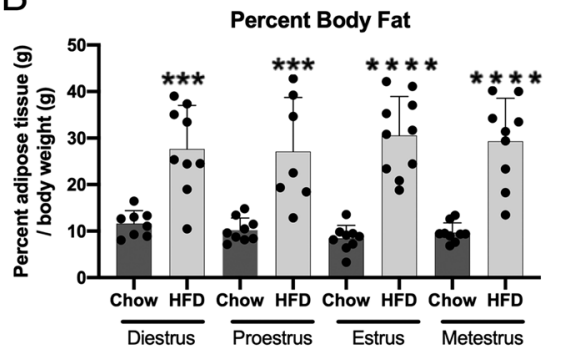

E

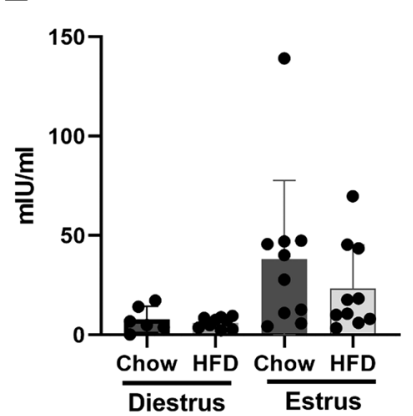

C

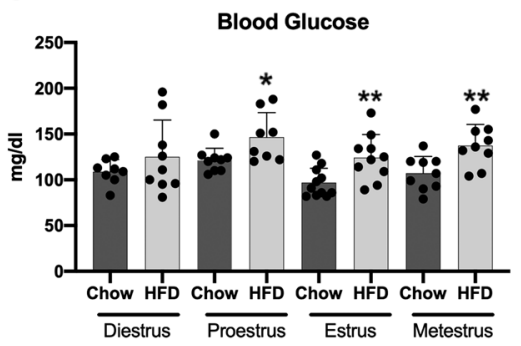

LH

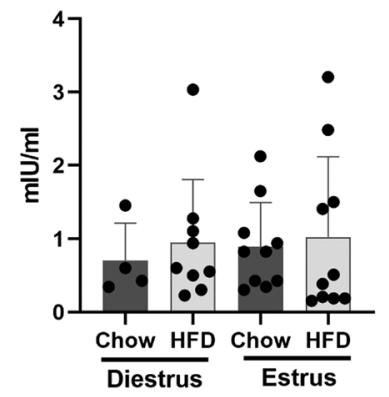

Figure 4 Impact of HFD on anthropometrics, estrous cyclicity, and FSH and LH levels. Anthropometric (A and B) and metabolomic (C) parameters were measured in mice after 10 weeks of HFD-exposure. Data are presented as means \pm S.D. Differences between groups was determined with a two-way ANOVA followed by Sidak's post hoc test for comparisons within diet and Tukey's post hoc test for comparisons within estrous stage if the overall ANOVA reached statistical significance. Daily vaginal cytology was assessed for the last 2 weeks of dietary feeding to evaluate the estrous cycle (D). Differences in estrous cyclicity between groups were determined by a Fisher's exact test. FSH and LH levels were measured in the serum during diestrus and estrus (E, F). Differences in FSH and LH were determined using Mann-Whitney test. ${ }^{*} P<$ $0.05 ;{ }^{* *} P<0.005 ;{ }^{* * *} P<0.0005 ;{ }^{* * * *} P<0.00005 . n=8-11$ group. Publicly available DOI for Figshare data: 10.6084/m9.figshare.12170124.

metestrus in our naturally cycle chow-fed mice. In the chow controls, we observed this expected pattern of Edn2 expression (Fig. 5A), aligning with the published data using superovulation models. It is important to note that we observed high variation in estrus in the chow fed mice. As ovulation occurs in the beginning of estrus, some ovaries were likely collected in estrus after ovulation had already occurred. In the HFD-fed mice, we observed a completely dysregulated pattern of ovarian Edn2 expression (Fig. 5B). Instead of observing a peak of Edn2 expression during estrus, we detected the lowest levels of ovarian Edn2 in estrus. Further, there
A
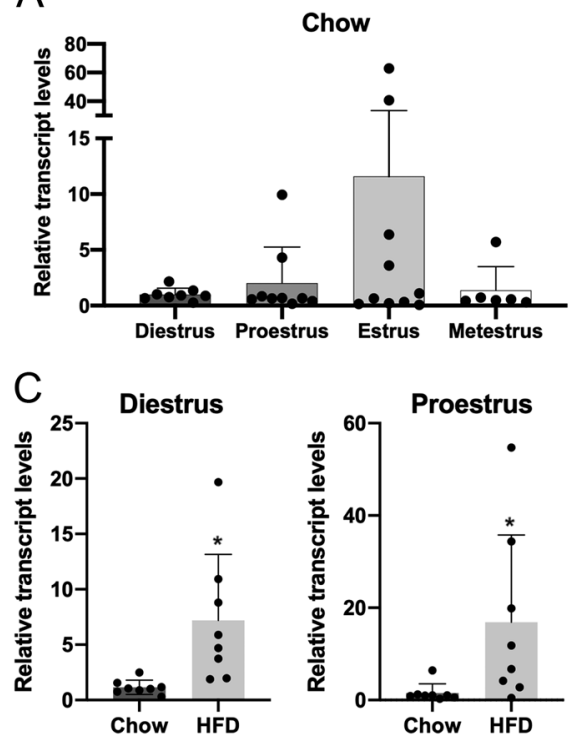

B

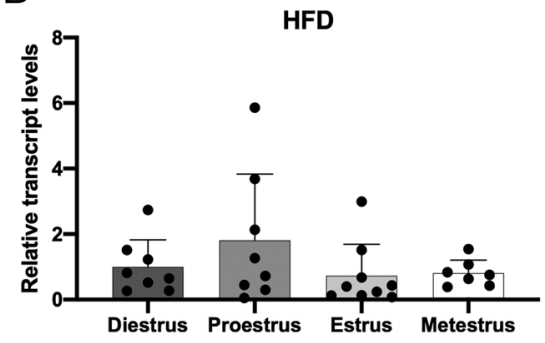

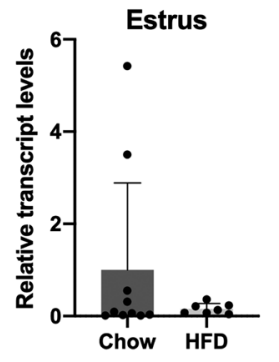

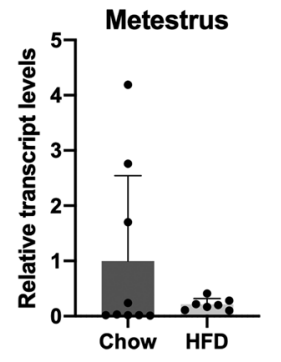

Figure 5 Ovarian Edn2 expression across the estrous cycle. After 10 weeks of dietary intervention, ovarian transcript levels of $E d n 2$ were determined (A, B and C). The expression profile of ovarian $E d n 2$ expression was first characterized across all stages of the estrous cycle in naturally cycling chow-fed controls (A) and then in the HFD-fed mice (B). Ovarian Edn2 expression levels were then compared within each estrous cycle stage between diets (C). Data are presented as means \pm S.D. Differences between groups were determined with a one-way ANOVA followed by Tukey's post hoc test if the overall ANOVA reached statistical significance (A and B) or a twotailed Student's $t$-test (C). ${ }^{*} P<0.05 . n=8-11$ / group. 
A

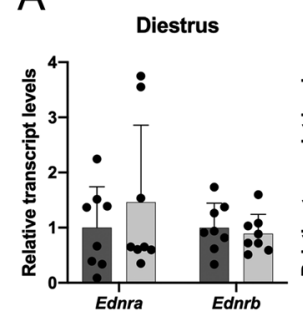

B

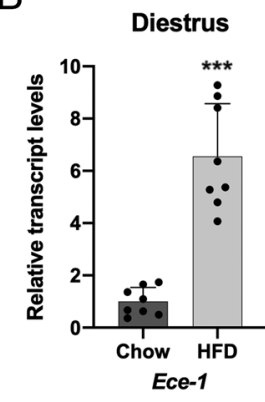

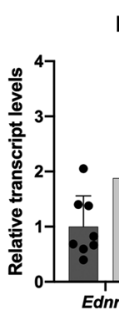

Proestrus
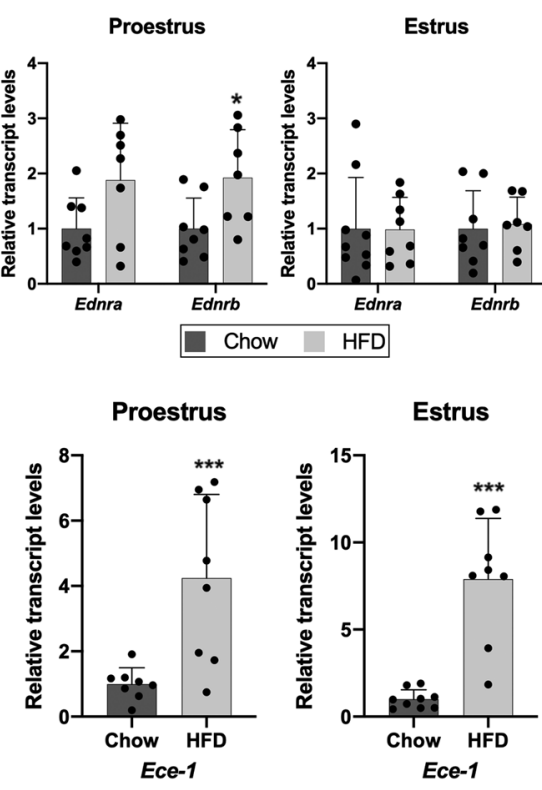

was little variation of Edn2 expression in the HFD mice with a less than two-fold increase in Edn2 observed in any stage of the estrous cycle. This is in sharp contrast to the chow mice, which had over a ten-fold increase in Edn2 levels during estrus.

We next examined ovarian Edn2 transcript levels comparing HFD-fed mice to chow-fed controls in each of the four estrous cycle stages. In each stage of the estrous cycle, Edn2 levels were dysregulated in the HFDfed mice (Fig. 5C). In both diestrus and proestrus, Edn2 was significantly increased in the HFD mice compared to the chow controls $(P<0.05)$. However, in estrus and metestrus, Edn2 was decreased compared to the chow controls, although these differences did not reach statistical significance $(P=0.26, P=0.20$, respectively), likely due to the high variability observed in the chow mice in these stages.

As we observed dysregulation of ovarian Edn2 with HFD, we next examined the expression of the other genes involved in ovarian ET2's actions in the ovary, the Edn2 receptors, endothelin receptor a (Ednra mRNA; ETA protein) and endothelin receptor b (Ednrb mRNA, ETB protein), and endothelin converting enzyme 1 (Ece1), which cleaves the ET2 peptide to its bioactive form (Ling et al. 2013) and has been suggested to be the Ece responsible for processing ET2 in the ovary (Bridges et al. 2010). Ednrb expression was increased in the HFD-fed mice compared to the chow-fed controls in proestrus and metestrus, however, this only reached statistical significance in proestrus (Fig. 6A). There were no differences in Ednra in the HFD-fed mice across the estrous cycle (Fig. 6A). In all four stages of the estrous cycle, Ece1 levels were significantly increased in the HFD-fed mice compared to the chow-fed controls (Fig. 6B, $P<0.05)$.
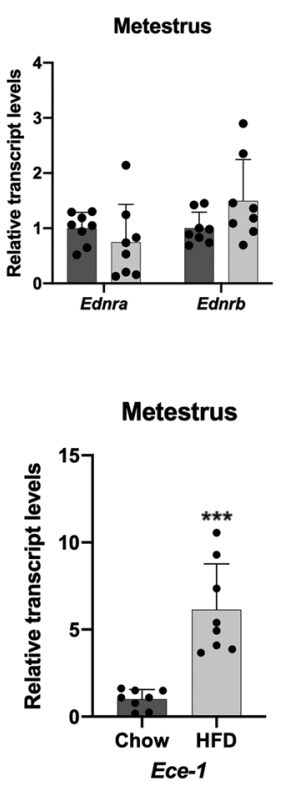

Figure 6 Impact of HFD on expression of genes involved in ET2 ovarian function. Ovarian transcripts of the endothelin receptors (A) and endothelin converting enzyme 1 (B) were determined across all stages of the estrous cycle after 10 weeks of dietary intervention. Data are presented as means \pm S.D. Differences between groups were determined by a two-tailed Student's $t$-test. ${ }^{*} P$ $<0.05,{ }^{* * *} P<0.0005 . n=8-11$ /group.

\section{Effect of HFD on ovarian steroidogenic gene expression}

We were interested in determining if there were any alterations in ovarian steroidogenic gene expression or in the estrogen receptors that may correspond with the HFD-dysregulated Edn2 data. After 10 weeks of HFD the ovarian expression of hydroxysteroid (17beta) dehydrogenase $1(H s d 17 b 1)$ was increased during proestrus in the HFD-fed mice (Fig. 7B, $P<0.05$ ), but was no different that chow-fed controls in all other stages of the estrous cycle (Fig. 7). Ovarian steroidogenic acute regulatory protein (Star) expression was increased in diestrus in the HFD-fed mice (Fig. 7A, $P<0.05$ ). There were no changes in the expression of cytochrome P450, family 11, subfamily a, polypeptide 1 (Cyp 11a1) in the HFD-fed mice (Fig. 7C). Cytochrome P450, family 19, subfamily a, polypeptide 1 (Cyp19a1) was only altered in metestrus, with increased expression in the HFDfed mice compared to the chow-fed controls (Fig. 7D, $P<0.05)$. Estrogen receptor alpha (Esr1) and estrogen receptor beta (Ers2) were significantly increased with HFD feeding in diestrus, proestrus, estrus, and metestrus (Fig. 7, $P<0.05$ ).

\section{Discussion}

In the current study, we used our previously established HFD mouse model to determine the potential involvement of dysregulation of ovarian Edn2 in HFDinduced ovulatory dysfunction. We showed that HFD feeding leads to an ovulatory defect independent of the development of obesity, and that ovarian Edn2 expression, which is critical for ovulation, is dysregulated across all stages of the estrous cycle, suggesting that 

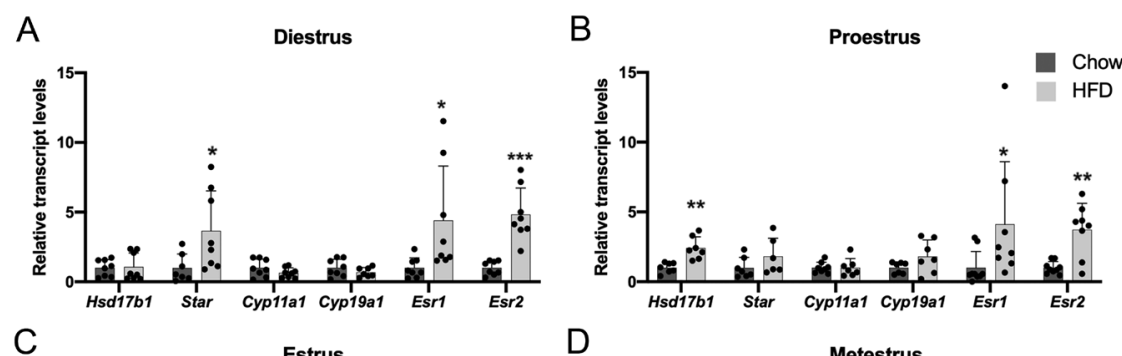

C

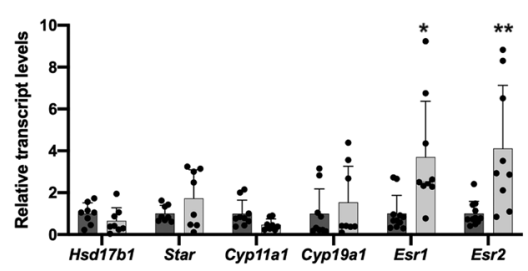

。

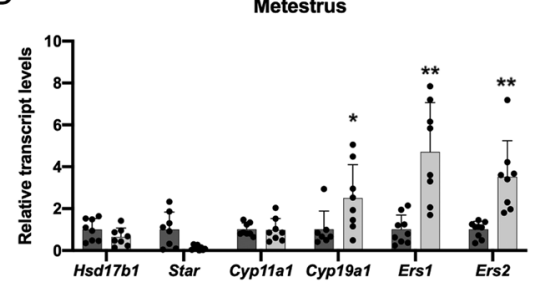

Figure 7 Impact of HFD on expression of ovarian steroidogenic genes and estrogen receptor transcripts. After 10 weeks of HFD transcripts of ovarian steroidogenic genes and the estrogen receptors were measured across all stages of the estrous cycle (A, B, C and D). Data are presented as means \pm S.D.

Differences were determined by a two-tailed Student's $t$-test. ${ }^{*} P<0.05 ;{ }^{* *} P<0.005 ;{ }^{* * *} P<$ 0.0005. $n=8-11$ /group. dysregulation of ovarian Edn2 may be involved in the observed ovulatory defect.

Interestingly, although we did observe fewer ovulated oocytes in both our HFOb and HFLn mice, the additional impact of obesity seemed to cause poorer oocyte development as a lower percentage of MII oocytes were collected from the HFOb mice after superovulation while the HFLn mice had a similar percentage of MII oocytes ovulated as the controls. This result was a bit surprising, as we have previously shown a similar ovarian phenotype (diminished ovarian reserve, abnormal estrous cycle) in regards to ovarian function in both lean and obese HFD-fed mice (Skaznik-Wikiel et al. 2016, Hohos et al. 2018). However, although we did observe fewer pups per litter in consecutive breeding attempts in both our lean and obese HFD-fed mice, this was more severe in the HFOb mice than in the HFLn mice (SkaznikWikiel et al. 2016). This more severe phenotype in the obese mice may be related to the fact that there are fewer developmentally mature oocytes in the HFD-fed obese mice than in the lean mice, translating to a more dramatic decrease in pups per litter than in the HFLn mice. Collectively, these data suggest that although the overt ovarian phenotype is negatively impacted equally in both the HFLn and HFOb mice, the actual maturation of the oocytes is impacted by HFD, but even more so when HFD is compounded with obesity.

Ovarian Edn2 has been shown to be critical for ovulation (Migone et al. 2016), thus dysregulation of ovarian Edn2 could lead to the observed HFD-induced ovulatory defect. Indeed, we saw dysregulation of ovarian $E d n 2$ expression across all stages of the estrous cycle in HFD-fed mice. What was most intriguing about the data was that the characteristic peak of Edn2 around the time ovulation was absent in the HFD-fed mice. In fact, in estrus, the HFD-fed mice had the lowest observed levels of ovarian Edn2. This missing peak of ovarian Edn2 could be responsible for the decreased number of ovulated oocytes in the HFD-fed mice. Considering that it has been shown that when ovarian ET2's action is blocked, there is a similar decrease in ovulated oocytes that was observed in our HFD-fed mice, however, ovulation is not entirely blocked (Ko et al. 2006), this seems like a plausible explanation. Future studies are needed to confirm the direct role of dysregulated ovarian Edn2 in HFD-induced ovulatory dysfunction.

As we observed dysregulated ovarian Edn2 expression with HFD feeding, we were interested to see if the expression of other genes involved in ET2's ovarian function was also dysregulated. Ovarian Ece 1 expression was increased across all stages of the estrous cycle in our HFD-fed mice. This upregulation of Ece 1 suggests that in addition to dysregulation of ovarian $E d n 2$ expression with HFD, the post-transcriptional processing of ET2 is also impaired, which could lead to preovulatory follicles getting the ET2 signal at the wrong time. Prior studies in the bovine model showed that Ece 1 is upregulated by IGF-1 and insulin (Levy et al. 2003). Although we did not measure insulin levels in our mice in the current study, both HFLn and HFOb mice had elevated fasting glucose levels. High-fat diet exposure has been shown to increase insulin levels and insulin resistance in mice in previous studies (Burcelin et al. 2002, Ahren et al. 2010, Kanno et al. 2015). Therefore, it is very plausible to assume that increased insulin levels in HFD mice were responsible for Ece1 upregulation throughout the estrous cycle in our study.

We also found some HFD-induced dysregulation of the endothelin receptors, particularly Ednrb. Some have reported that culturing ovarian strips only with the EDNRA antagonist but not the EDNRB antagonist prevents contraction when ET2 is given, suggesting that EDNRA is responsible for ET2 ovarian action. However, in in vivo follow up studies intra-ovarian injection of both antagonists did not impact ovulation (Bridges et al. 2010). Others have shown that ovarian injection of the dual ET2 receptor antagonist did inhibit ovulation (Ko et al. 2006) and injection of the EDNRA inhibitor decreased ovulation by $25 \%$ while injection of the EDNRB inhibitor decreased ovulated oocytes by $75 \%$ (Palanisamy et al. 
2006), suggesting that both receptors may be involved in facilitating ET2's action and that EDNTB may play a larger role. However, Cho et al. showed in EDNRB-KO female mice that EDNRB is not necessary for ovulation to occur. EDNRB-KO mice ovulated normally but had more corpora lutea and larger litter sizes, indicating that EDNRB is also necessary to limit ovulation (Cho et al. 2012). Considering we saw more of an impact of Ednrb expression with HFD feeding, perhaps this is the more important receptor, however, the ovulatory defect could also be due to the overall lack of Edn2 in HFD-fed mice. More work is needed to elucidate the role of the endothelin receptors in ovarian ET2 signaling.

Progesterone (Palanisamy et al. 2006, Breen et al. 2013, Tsubota et al. 2015), hypoxia (Zhang et al. 2010, Yalu et al. 2015, Migone et al. 2016), and LH (Klipper et al. 2010, Yalu et al. 2015) have all been suggested as potential upstream regulators of ovarian ET2, however, there is little consensus on what actually is regulating ovarian Edn2 and many possible upstream regulators have yet to be considered. Cell cultures of granulosa cells have shown that both $\mathrm{LH}$ and hypoxia increase Edn2 expression (Klipper et al. 2010). It has been proposed that Hif1a, a hypoxia factor, is increased in the corpus luteum and induces the expression of $E d n 2$, supporting its role as a luteinization gene (Yalu et al. 2015) . Moreover, $\mathrm{Na}$ et al. showed that Hif1a is a transcription factor for Edn2 expression and Edn2 is one of the HIF target genes in the ovary (Na et al. 2008). The HIF-1alpha induced production of ET2 is likely to promote vascular changes that facilitate the ovulatory process (Palanisamy et al. 2006, Kim et al. 2009). Interestingly, HIF-1alpha activity has been shown to be increased in adipose tissue secondary to hypoxia and has been found to contribute to chronic inflammation in obesity (He et al. 2011, Boutens et al. 2018). Although not all the mice fed HFD in our study became obese, we noticed that HFD feeding was associated with increased lipid deposition in the ovaries, regardless of body composition. Perhaps, HIF-1alpha is dysregulated in HFD feeding which results in subsequent $E d n 2$ dysregulation.

We were interested to see if there were any alterations in ovarian steroidogenic genes and/or the estrogen receptor transcripts in the ovaries of HFD-fed mice, which may be related to the decreased $E d n 2$ expression. The most interesting changes observed in ovarian steroidogenic genes by HFD were Star and Cyp19a1. Interestingly, both ovarian Star and Cyp19a1 are regulated by increased protein kinase $A$ (PKA) activity (Jamnongjit \& Hammes 2006), suggesting a possible upstream regulator for the observed dysregulated gene expression. Further, Star is the rate limiting step in ovarian steroidogenesis (Jamnongjit \& Hammes 2006), suggesting that there may be larger implications to ovarian steroid metabolism with HFD exposure. The increased expression of Cyp19a1 and the increased expression of both the estrogen receptors across all stages of the estrous cycle suggest impaired estrogen action in the ovary Future work is needed to further elucidate the role of HFD-induced impaired ovarian steroidogenesis in ovarian Edn2 expression and ovulation.

Several limitations to our work do restrict the translatability of the findings and highlight areas for additional work. First, whole ovaries were used rather than isolated cells which could interfere with our interpretation of the findings. Second, we have focused on ovulatory dysfunction in this model and have not considered in detail the other aspects of the female hypothalamuspituitary axis, which is critical for correct functioning of the female reproduction system. In this study, we did not observe any differences in either $\mathrm{LH}$ or FSH between our HFD and chow-fed mice, however, the estrous cycle was assessed by vaginal smears therefore accurate assessment of the LH surge could not be ascertained. LH levels remain low throughout the entire estrous cycle and the exact surge is difficult to measure in naturally cycling mice. Therefore, future work is needed to fully elucidate the role of the hypothalamus and pituitary along with the ovary in HFD-induced reproductive dysfunction by challenging animals with $\mathrm{GnRH}$ or Kisspeptin before measuring LH. Finally, we utilized naturally cycling mice for this study as it is critical to understand endothelin-2's role in ovulation in a natural model in addition to the superovulated models that have been exclusively used. However, as we did not synchronize cyclicity with exogenous hormones, the ovaries collected from mice in each estrous stage as determined by vaginal cytology will not all be from mice in the exact same stage of each estrous cycle. This is particularly impactful in ovaries collected from mice in estrus, as some may have already ovulated, be periovulatory, or pre-ovulatory. To overcome the limitation of potentially inaccurate cycle assessment, future studies are needed to confirm that an altered $E d n 2$ expression is indeed responsible for decreased ovulation in HFD mice by challenging the animals with $\mathrm{LH}$ or $\mathrm{GnRH} / \mathrm{Kisspeptin}$ before measuring Edn2 levels.

In conclusion, HFD feeding causes impaired ovulation independent of the development of obesity in female mice and dysregulation of ovarian Edn2 expression across all stages of the estrous cycle. These data suggest that dysregulation of ovarian Edn2 may be involved in the observed HFD-induced ovulatory defect, which may in part be due to impaired estradiol signaling.

\section{Declaration of interest}

The authors declare that there is no conflict of interest that could be perceived as prejudicing the impartiality of the research reported.

\section{Funding}

This work was supported by the Colorado NORC Pilot Grant (P30DK048520) and the University of Colorado's Building 
Interdisciplinary Research Careers in Women's Health (BIRCWH) Grant (K12 HD057022) to M E S-W.

\section{Author contribution statement}

$\mathrm{N} \mathrm{M} \mathrm{H} \mathrm{participated} \mathrm{in} \mathrm{the} \mathrm{design} \mathrm{of} \mathrm{the} \mathrm{work,} \mathrm{data} \mathrm{collection,}$ analysis and interpretation, and drafting and revising the work. E M E, A G, E S and J D R participated in the acquisition of data and revising the work. ME S-W participated in the conception and design of the work, the interpretation of data, and drafting and revising the manuscript.

\section{References}

Ahren J, Ahren B \& Wierup N 2010 Increased beta-cell volume in mice fed a high-fat diet: a dynamic study over 12 months. Islets 2 353-356. (https://doi.org/10.4161/isl.2.6.13619)

Boutens L, Hooiveld GJ, Dhingra S, Cramer RA, Netea MG \& Stienstra R 2018 Unique metabolic activation of adipose tissue macrophages in obesity promotes inflammatory responses. Diabetologia 61 942-953. (https://doi.org/10.1007/s00125-017-4526-6)

Breen SM, Andric N, Ping T, Xie F, Offermans S, Gossen JA \& Ascoli M 2013 Ovulation involves the luteinizing hormone-dependent activation of G(q/11) in granulosa cells. Molecular Endocrinology 27 1483-1491. (https://doi.org/10.1210/me.2013-1130)

Bridges PJ, Jo M, Al Alem L, Na G, Su W, Gong MC, Jeoung M \& Ko C 2010 Production and binding of endothelin-2 (EDN2) in the rat ovary: endothelin receptor subtype A (EDNRA)-mediated contraction. Reproduction, Fertility, and Development 22 780-787. (https://doi. org/10.1071/RD09194)

Burcelin R, Crivelli V, Dacosta A, Roy-Tirelli A \& Thorens B 2002 Heterogeneous metabolic adaptation of $\mathrm{C} 57 \mathrm{BL} / 6 \mathrm{~J}$ mice to high-fat diet. American Journal of Physiology: Endocrinology and Metabolism 282 E834-E842. (https://doi.org/10.1152/ajpendo.00332.2001)

Cacioppo JA, Oh SW, Kim HY, Cho J, Lin PC, Yanagisawa M \& Ko C 2014 Loss of function of endothelin-2 leads to reduced ovulation and CL formation. PLOS ONE 9 e96115. (https://doi.org/10.1371/journal. pone.0096115)

Cacioppo JA, Koo Y, Lin PC, Gal A \& Ko C 2015 Generation and characterization of an endothelin-2 iCre mouse. Genesis 53 245-256. (https://doi.org/10.1002/dvg.22845)

Cacioppo JA, Lin PP, Hannon PR, Mcdougle DR, Gal A \& Ko C 2017 Granulosa cell endothelin-2 expression is fundamental for ovulatory follicle rupture. Scientific Reports 7 817. (https://doi.org/10.1038/ s41598-017-00943-w)

Caligioni CS 2009 Assessing reproductive status/stages in mice. Current Protocols in Neuroscience $\mathbf{4 8}$ Appendix 41.1-41.8. (https://doi. org/10.1002/0471142301.nsa04is48)

Cho J, Kim H, Kang DW, Yanagisawa M \& Ko C 2012 Endothelin B receptor is not required but necessary for finite regulation of ovulation. Life Sciences 91 613-617. (https://doi.org/10.1016/j.Ifs.2012.02.016)

Choi DH, Kim EK, Kim KH, Lee KA, Kang DW, Kim HY, Bridges P \& Ko C 2011 Expression pattern of endothelin system components and localization of smooth muscle cells in the human pre-ovulatory follicle. Human Reproduction 26 1171-1180. (https://doi.org/10.1093/humrep/ der066)

Choudhary E, Costine BA, Wilson ME, Inskeep EK \& Flores JA 2004 Prostaglandin F2alpha (PGF2alpha) independent and dependent regulation of the bovine luteal endothelin system. Domestic Animal Endocrinology 27 63-79. (https://doi.org/10.1016/j.domaniend.2004.02.001)

Costine BA, Inskeep EK, Blemings KP, Flores JA \& Wilson ME 2007 Mechanisms of reduced luteal sensitivity to prostaglandin F2alpha during maternal recognition of pregnancy in ewes. Domestic Animal Endocrinology 32 106-121. (https://doi.org/10.1016/j. domaniend.2006.01.003)

Ge ZJ, Luo SM, Lin F, Liang QX, Huang L, Wei YC, Hou Y, Han ZM, Schatten H \& Sun QY 2014 DNA methylation in oocytes and liver of female mice and their offspring: effects of high-fat-diet-induced obesity. Environmental Health Perspectives 122 159-164. (https://doi. org/10.1289/ehp.1307047)

He Q, Gao Z, Yin J, Zhang J, Yun Z \& Ye J 2011 Regulation of HIF-1\{alpha\} activity in adipose tissue by obesity-associated factors: adipogenesis, insulin, and hypoxia. American Journal of Physiology: Endocrinology and Metabolism $300 \quad$ E877-E885. (https://doi.org/10.1152/ ajpendo.00626.2010)

Hohos NM, Cho KJ, Swindle DC \& Skaznik-Wikiel ME 2018 High-fat diet exposure, regardless of induction of obesity, is associated with altered expression of genes critical to normal ovulatory function. Molecular and Cellular Endocrinology 470 199-207. (https://doi.org/10.1016/j. mce.2017.10.016)

Jamnongjit M \& Hammes SR 2006 Ovarian steroids - the good, the bad, and the signals that raise them. Cell Cycle 5 1178-1183. (https://doi. org/10.4161/cc.5.11.2803)

Kanno A, Asahara SI, Masuda K, Matsuda T, Kimura-Koyanagi M, Seino S, Ogawa W \& Kido Y 2015 Compensatory hyperinsulinemia in high-fat diet-induced obese mice is associated with enhanced insulin translation in islets. Biochemical and Biophysical Research Communications 458 681-686. (https://doi.org/10.1016/j.bbrc.2015.02.024)

Kim J, Bagchi IC \& Bagchi MK 2009 Signaling by hypoxia-inducible factors is critical for ovulation in mice. Endocrinology 150 3392-3400. (https:// doi.org/10.1210/en.2008-0948)

Klipper E, Levit A, Mastich Y, Berisha B, Schams D \& Meidan R 2010 Induction of endothelin-2 expression by luteinizing hormone and hypoxia: possible role in bovine corpus luteum formation. Endocrinology 151 1914-1922. (https://doi.org/10.1210/en.2009-0767)

Ko C, Gieske MC, Al-Alem L, Hahn Y, Su W, Gong MC, Iglarz M \& Koo Y 2006 Endothelin-2 in ovarian follicle rupture. Endocrinology 147 1770-1779. (https://doi.org/10.1210/en.2005-1228)

Levy N, Gordin M, Smith MF, Bolden-Tiller OU \& Meidan R 2003 Hormonal regulation and cell-specific expression of endothelinconverting enzyme 1 isoforms in bovine ovarian endothelial and steroidogenic cells. Biology of Reproduction 68 1361-1368. (https://doi. org/10.1095/biolreprod.102.009134)

Ling L, Maguire JJ \& Davenport AP 2013 Endothelin-2, the forgotten isoform: emerging role in the cardiovascular system, ovarian development, immunology and cancer. British Journal of Pharmacology 168 283-295. (https://doi.org/10.1111/j.1476-5381.2011.01786.x)

Livak KJ \& Schmittgen TD 2001 Analysis of relative gene expression data using real-time quantitative PCR and the 2(T)(-Delta Delta C) method. Methods 25 402-408. (https://doi.org/10.1006/meth.2001.1262)

Mao L, Lou H, Lou Y, Wang N \& Jin F 2014 Behaviour of cytoplasmic organelles and cytoskeleton during oocyte maturation. Reproductive Biomedicine Online 28 284-299. (https://doi.org/10.1016/j. rbmo.2013.10.016)

Migone FF, Cowan RG, Williams RM, Gorse KJ, Zipfel WR \& Quirk SM 2016 In vivo imaging reveals an essential role of vasoconstriction in rupture of the ovarian follicle at ovulation. PNAS 113 2294-2299. (https://doi.org/10.1073/pnas.1512304113)

Na G, Bridges PJ, Koo Y \& Ko C 2008 Role of hypoxia in the regulation of periovulatory EDN2 expression in the mouse. Canadian Journal of Physiology and Pharmacology 86 310-319. (https://doi.org/10.1139/ Y08-025)

Palanisamy GS, Cheon YP, Kim J, Kannan A, Li Q, Sato M, Mantena SR, Sitruk-Ware RL, Bagchi MK \& Bagchi IC 2006 A novel pathway involving progesterone receptor, endothelin-2, and endothelin receptor B controls ovulation in mice. Molecular Endocrinology 20 2784-2795. (https://doi. org/10.1210/me.2006-0093)

Shimizu T, Berisha B, Schams D \& Miyamoto A 2007 Changes in the messenger RNA expressions of the endothelin-1 and angiotensin systems in mature follicles of the superovulated bovine ovary. Journal of Reproduction and Development 53 655-662. (https://doi.org/10.1262/ jrd.18168)

Skaznik-Wikiel ME, Swindle DC, Allshouse AA, Polotsky AJ \& Mcmanaman JL 2016 High-fat diet causes subfertility and compromised ovarian function independent of obesity in mice. Biology of Reproduction 94 108. (https://doi.org/10.1095/biolreprod.115.137414)

Tsubota K, Kanki M, Noto T, Nakatsuji S, Oishi Y, Matsumoto M \& Nakayama H 2015 Altered gene expression profile in ovarian follicle 
in rats treated with indomethacin and RU486. Journal of Toxicological Sciences 40 413-425. (https://doi.org/10.2131/jts.40.413)

Wang Z, Zhang Z, Wu Y, Chen L, Luo Q, Zhang J, Chen J, Luo Z, Huang X \& Cheng Y 2012 Effects of echinomycin on endothelin-2 expression and ovulation in immature rats primed with gonadotropins. Experimental and Molecular Medicine 44 615-621. (https://doi.org/10.3858/ emm.2012.44.10.070)

Wang LY, Wang N, Le F, Li L, Lou HY, Liu XZ, Zheng YM, Qian YQ, Chen YL, Jiang XH, et al. 2015 Superovulation induced changes of lipid metabolism in ovaries and embryos and its probable mechanism. PLoS ONE 10 e0132638. (https://doi.org/10.1371/journal.pone.0132638)

Yalu R, Oyesiij AE, Eisenberg I, Imbar T \& Meidan R 2015 HIF1A-dependent increase in endothelin 2 levels in granulosa cells: role of hypoxia, LH/ cAMP, and reactive oxygen species. Reproduction 149 11-20. (https:// doi.org/10.1530/REP-14-0409)

Yang X, Dunning KR, Wu LL, Hickey TE, Norman RJ, Russell DL, Liang X \& Robker RL 2010 Identification of perilipin-2 as a lipid droplet protein regulated in oocytes during maturation. Reproduction, Fertility, and Development 22 1262-1271. (https://doi.org/10.1071/RD10091)
Zhang Y, Diao Z, Su L, Sun H, Li R, Cui H \& Hu Y 2010 MicroRNA-155 contributes to preeclampsia by down-regulating CYR61. American Journal of Obstetrics and Gynecology 202 466.e1-466.e7. (https://doi. org/10.1016/j.ajog.2010.01.057)

Zhang J, Zhang Z, Wu Y, Chen L, Luo Q, Chen J, Huang X, Cheng Y \& Wang Z 2012 Regulatory effect of hypoxia-inducible factor-1alpha on hCG-stimulated endothelin-2 expression in granulosa cells from the PMSG-treated rat ovary. Journal of Reproduction and Development $\mathbf{5 8}$ 678-684. (https://doi.org/10.1262/jrd.2012-089)

Received 26 May 2020

First decision 29 June 2020

Revised manuscript received 3 December 2020

Accepted 11 January 2021 